\title{
The biology and the importance of Photobacterium species
}

\begin{abstract}
Photobacterium species are Gram-negative coccobacilli which are distributed in marine habitats worldwide. Some species are unique because of their capability to produce luminescence. Taxonomically, about 23 species and 2 subspecies are validated to date. Genomes from a few Photobacterium spp. have been sequenced and studied. They are considered a special group of bacteria because some species are capable of producing essential polyunsaturated fatty acids, antibacterial compounds, lipases, esterases and asparaginases. They are also used as biosensors in food and environmental monitoring and detectors of drown victim, as well as an important symbiont.
\end{abstract}

Keyword: Applications; Genome; Marine bacteria; Photobacterium 